\title{
Dysregulation of gut microbiota and chronic inflammatory disease: from epithelial defense to host immunity
}

Experimental \& Molecular Medicine (2017) 49, e337; doi:10.1038/emm.2017.55; published online 26 May 2017

$\mathrm{T}$ he mammalian microbiota is a community of commensal microorganisms including bacteria, archaea, eukaryotes and viruses. Microbial colonization begins at birth. ${ }^{1}$ During infancy, the microbial community shifts chaotically in response to dietary changes, illness and antibiotics. ${ }^{2}$ The abundance and diversity of the microbiota reach adult levels in early childhood, and the core composition is thereafter considered to remain stable for decades. ${ }^{3}$

The microbiome is the genomic collection of the entire repertoire microbiota. The gastrointestinal tract is the largest habitat of microbiota. ${ }^{4}$ Thus, culture-based methods are limited in their potential to investigate the composition and physiological/ecological functions of the microbiota. Currently, data on the microbiome are obtained mainly by two methods: (1) sequencing of $16 \mathrm{~S}$ ribosomal RNA (rRNA) genes, which is used to classify microbial communities, and (2) shotgun-sequencing techniques (including meta-genomic and meta-transcriptomic analyses), which provide information on the functions and activity of the microbiome. ${ }^{5}$ The $16 \mathrm{~S}$ rRNA gene contains both conserved sequences that can be targeted by universal bacterial PCR primers and hypervariable regions that can be used to identify bacterial phylotypes. ${ }^{6}$ These methods permit a relatively unbiased characterization of microbial diversity in various environments. ${ }^{5}$ However, 16S rRNA sequences alone do not provide information regarding function. The second widely used method, whole-genome shotgun next-generation sequencing (NGS), makes it possible to generate functional profiles via DNA-focused meta-genomic and RNA-focused meta-transcriptomic analyses. ${ }^{7}$ Two large-scale studies using NGS, the European Meta-genomics of the Human Intestinal Tract $(\text { MetaHIT })^{8}$ and the US Human Gut Microbiome Project (HMP), ${ }^{9}$ provide baseline data on the composition and function of the human microbiome. In addition, simultaneous sequencing of the metagenome and metatranscriptome of the host and microbiota provides insight into mechanisms of host-microbiota mutualism and can be used to differentiate between unhealthy and healthy individuals. ${ }^{10}$ More recently, metabolomic and meta-proteomic strategies have been applied to host-microbiome studies. ${ }^{11}$

In the intestinal lumen, the microbiota is consistently monitored by the mucosal immune system. Although the intestinal epithelial cells (IECs) are not traditionally classified as cells of innate immune response, they express an extensive repertoire of pattern-recognition receptors, such as Toll-like receptors. ${ }^{12}$ Moreover, microbiota-induced signal transduction in IECs is crucial for maintaining the balance between tolerance to commensal microbes and elimination of pathogens. ${ }^{13}$ The IECs also serve as a physical and chemical barrier between the mucosa and microbiota by forming a mucus layer, secreting antimicrobial peptides, and regenerating the islet-derived protein family. In addition to their barrier function, IECs serve as 'mediators', maintaining a balanced relationship between gut microbes and the host immune system by secreting cytokines, chemokines and hormones. Thus, dysfunction of the IECs leads to perturbation of the gut microbiota and enhances susceptibility to intestinal inflammation (see review by Okumura and Takeda in this issue).

The crosstalk between the gut microbiota and the host immune system begins at birth. The immune system induces and maintains tolerance to autochthonous biota. At the same time, the immune system initiates an effective immune response against commensals, pathobionts (microbes that are usually symbionts but are also capable of causing pathology under certain conditions) and external pathogens. ${ }^{14}$ In turn, the microbiota modulates the development and function of innate and adaptive immunity, including peripheral differentiation of T-helper cells. Co-production of metabolites by the host and gut microbiota as they metabolize food and xenobiotics involves these immune-mediated signaling processes. ${ }^{15}$ For example, short-chain fatty acids (SCFAs) are important energy sources for both the gut microbiota and IECs. SCFAs also influence macrophages and peripheral $\mathrm{T}$ cells, particularly regulatory $\mathrm{T}$ cells (see reviews by Lee and Kim and by Kim et al. in this issue). ${ }^{16}$ 
Dysbiosis is a state of imbalance in the composition or function of microbial taxa. Basic and applied research on dysbiosis related to human disease has greatly advanced in recent decades. Various chronic diseases including inflammatory bowel disease, multiple sclerosis, rheumatoid arthritis, asthma, diabetes and obesity are associated with dysbiosis in the gut (see review by Lee and Kim, and review by Kim et al. in this issue). As our understanding of the role of dysbiosis in the pathogenesis of chronic diseases has improved, the maintenance of a healthy microbiota has come to be considered as a new therapeutic target. The restoration of microbiotic homeostasis is thought to be an effective therapeutic approach to attenuating dysbiosis-induced inflammation and promoting disease remission. Modalities employed include probiotics (live microorganisms that when ingested provide benefit to the host), bacteriophages (phages that specifically infect and kill certain bacterial species) and fecal microbiota transplantation (see review by Kim et al. in this issue).

This themed issue includes three review articles describing how IECs are mediated between the gut microbiota and the host, and how dysbiosis is linked to various diseases. The maintenance of a healthy microbiota has potential as an approach to developing effective therapeutic strategies and promoting health in some individuals. Over the last decade, our understanding of how the gut microbiota contributes to host health has progressed greatly. In the coming decade, this field of research is expected to reach milestones with diagnostic, preventive and therapeutic applications.

\section{CONFLICT OF INTEREST}

The authors declare no conflict of interest.

\section{ACKNOWLEDGEMENTS}

This work was supported by grants from the National Research Foundation of Korea (NRF) funded by the Ministry of Education, Science and Technology (2015R1A3A2032927 and 2016R1D1A1A02937326).

Jung Hee $\mathrm{Koh}^{1,2}$ and Wan-Uk Kim ${ }^{1,2}$ ${ }^{1}$ Center for Integrative Rheumatoid Transcriptomics and Dynamics, College of Medicine, The Catholic University of Korea, Seoul, Korea and ${ }^{2}$ Division of Rheumatology, Department of Internal Medicine, Seoul St. Mary's Hospital, College of Medicine, The Catholic University of Korea, Seoul, Korea E-mail:wan725@catholic.ac.kr
1 Dominguez-Bello MG, Costello EK, Contreras M, Magris M, Hidalgo G, Fierer $\mathrm{N}$ et al. Delivery mode shapes the acquisition and structure of the initial microbiota across multiple body habitats in newborns. Proc Natl Acad Sci USA 2010; 107: 11971-11975.

2 Koenig JE, Spor A, Scalfone N, Fricker AD, Stombaugh J, Knight R et al. Succession of microbial consortia in the developing infant gut microbiome. Proc Natl Acad Sci USA 2011; 108 (Suppl 1): 4578-4585.

3 Faith JJ, Guruge JL, Charbonneau M, Subramanian S, Seedorf H, Goodman $A L$ et al. The long-term stability of the human gut microbiota. Science 2013; 341: doi: 10.1126/science.1237439.

4 Spor A, Koren O, Ley R. Unravelling the effects of the environment and host genotype on the gut microbiome. Nat Rev Microbiol 2011; 9: 279-290.

5 Goodrich JK, Di Rienzi SC, Poole AC, Koren O, Walters WA, Caporaso JG et al. Conducting a microbiome study. Cell 2014; 158: 250-262.

6 Weisburg WG, Barns SM, Pelletier DA, Lane DJ. 16S ribosomal DNA amplification for phylogenetic study. J Bacteriol 1991; 173: 697-703.

7 Morgan XC, Huttenhower C. Meta'omic analytic techniques for studying the intestinal microbiome. Gastroenterology 2014; 146: 1437-1448 e1431.

8 Qin J, Li R, Raes J, Arumugam M, Burgdorf KS, Manichanh C et al. A human gut microbial gene catalogue established by metagenomic sequencing. Nature 2010; 464: 59-65.

9 Peterson J, Garges S, Giovanni M, Mclnnes P, Wang L, Schloss JA et al. The NIH Human Microbiome Project. Genome Res 2009; 19: 2317-2323.

10 Franzosa EA, Hsu T, Sirota-Madi A, Shafquat A, Abu-Ali G, Morgan XC et al. Sequencing and beyond: integrating molecular 'omics' for microbial community profiling. Nat Rev Microbiol 2015; 13: 360-372.

11 Sridharan GV, Choi K, Klemashevich C, Wu C, Prabakaran D, Pan LB et al. Prediction and quantification of bioactive microbiota metabolites in the mouse gut. Nat Commun 2014; 5: 5492.

12 Rakoff-Nahoum S, Paglino J, Eslami-Varzaneh F, Edberg S, Medzhitov R. Recognition of commensal microflora by toll-like receptors is required for intestinal homeostasis. Cell 2004; 118: 229-241.

13 Thaiss CA, Zmora N, Levy M, Elinav E. The microbiome and innate immunity. Nature 2016; 535: 65-74.

14 Palm NW, de Zoete MR, Flavell RA. Immune-microbiota interactions in health and disease. Clin Immunol 2015; 159: 122-127.

15 Nicholson JK, Holmes E, Kinross J, Burcelin R, Gibson G, Jia W et al. Host-gut microbiota metabolic interactions. Science 2012; 336: 1262-1267.

16 Rooks MG, Garrett WS. Gut microbiota, metabolites and host immunity. Nat Rev Immunol 2016; 16: 341-352.

(c) (i) $(-)$ This work is licensed under a Creative Commons Attribution-NonCommercial-NoDerivs 4.0 International License. The images or other third party material in this article are included in the article's Creative Commons license, unless indicated otherwise in the credit line; if the material is not included under the Creative Commons license, users will need to obtain permission from the license holder to reproduce the material. To view a copy of this license, visit http://creativecommons.org/licenses/by-nc-nd/4.0/ 